\title{
RESEARCH
}

IN BRIEF

- This paper will facilitate future definition of what is involved in assessment prior to chair dental general anaesthesia (CDGA).

- It will prompt some debate about the location of assessment services in relation to the CDGA service and to conscious sedation alternatives.

- It will prompt discussion about the level of training and experience required by the dental 'assessors', should they be specialist paediatric dentists?

- This paper might also prompt further guideline development in relation to the CGDA pathway of care.

\section{Assessment of children prior to dental extractions under general anaesthesia in Scotland}

\author{
C. Tochel, ${ }_{1}^{1}$ M.-T. Hosey, ${ }^{2}$ L. Macpherson ${ }^{3}$ and C. Pine ${ }^{4}$
}

\begin{abstract}
Objective To determine the type of dental assessment service offered to children prior to exodontia under chair dental general anaesthesia (CDGA) in the community and hospital dental services (CDS, HDS) in Scotland.

Design and setting Telephone interviews were conducted with CDS and HDS clinical directors in Scotland using a semi-structured questionnaire, with written follow-up confirmation. Copies of CDGA referral forms were also requested.

Results All clinical directors, or their nominated deputies, participated $(n=21)$. Almost half of interviewees indicated that their area offered a 'dedicated' pre-CDGA assessment service $(n=10)$. A range of grades was identified amongst assessors; a minority were described as 'specialist paediatric staff'. The availability of CDGA alternatives, including local analgesia and conscious sedation varied. Changes to the referral treatment plan were reported to occur 'sometimes' or 'often' by 11 interviewees regarding the anaesthetic used, and by 16 respondents regarding the number of teeth extracted. The content of CDGA referral forms was diverse.

Conclusions The method of referral and assessment of children for CDGA in Scotland is diverse, partly reflecting geographic limitations and local need. Guidance on pre-GA assessment, including use of standardised referral forms, may reduce variation and improve the quality of the pathway of care.
\end{abstract}

Recent guidance from the Scottish Executive Health Department has emphasised that chair dental general anaesthesia (CDGA) should be undertaken only where there is clinical need, and that all patients should be assessed by dental practitioners 'skilled and trained in the treatment of children'. ${ }^{1}$ Additionally,

${ }^{1}$ Research Assistant, ${ }^{2 *}$ Senior Lecturer/Honorary Consultant in Paediatric Dentistry, ${ }^{3}$ Senior Lecturer/Honorary Consultant in Dental Public Health, University of Glasgow Dental

School, 378 Sauchiehall Street, Glasgow, G2 3JZ; ${ }^{4}$ Professor of Dental Public Health and

Primary Dental Care, Department of Clinical Dental Sciences, Liverpool University Dental

Hospital and School of Dentistry, Pembroke Place,

Liverpool L3 5PS

${ }^{*}$ Correspondence to: Marie-Therese Hosey

Email:m.t.hosey@dental.gla.ac.uk

\section{Refereed Paper}

doi:10.1038/sj.bdj.4811281

Received 20.02.03; Accepted 01.07.03

๑) British Dental Journal 2004; 196: 629-633 it has been stated that parents should be informed of the risk of general anaesthesia and offered, where indicated, a range of alternative treatments. ${ }^{2}$ Therefore, a greater obligation has now been placed on the dental profession in respect of assessment, patient information and in obtaining informed consent.

In many community dental services (CDS) and hospital dental services (HDS), pre-GA assessment clinics have been set up separately from the CDGA visit. However, no national information is available in Scotland on how universal this approach has become. In some parts of England, pre-GA assessment clinics in the CDS have been used to reduce the number of patients receiving a CDGA. This approach has ensured that the anaesthetist has a satisfactory medical history and the operating dental surgeon has appropriate clinical information to undertake the proposed care. In an audit in Leicestershire, reviewing almost 600 referrals from the GDS to the CDS, for 35\% of cases, the original treatment request from the GDS was changed by the CDS and in $8 \%$ of cases, treatment was provided under a local anaesthetic. In only $45 \%$ of GA cases did extractions occur exactly as had been requested by the referring clinicians. Overall, the pre-GA assessment clinic in this audit prevented the need for GA in 15\% of referrals from general dental practitioners (GDPs). ${ }^{3}$

Studies have shown that referral to a specialist paediatric dentistry screening service leads to an increased number of extractions, resulting in a reduction in the need for a repeat GA. ${ }^{4,5}$ However, a study carried out in 1991 in a Scottish secondary centre reported that $40 \%$ of the children who received a GA had only one or two teeth extracted. ${ }^{6}$ The problem of attendance for repeat referral is a serious one, with reports of up to $31 \%$ of children requiring further GA. ${ }^{7}$ One reason for this need for a repeat CDGA for dental extractions could be incomplete caries diagnosis or deficient management of pre-existing caries at the time of the first referral.

Therefore, further reduction in the number of children undergoing CDGA might be achieved if the dental assessment procedure is more robust, but firstly there has to be a clearer understanding of the existing mechanism for pre-CDGA assessment. Therefore, the aim of this study was to collect information on the current provision of pre-CDGA dental assessment services in Scotland. 


\section{METHODS}

A short pilot questionnaire was sent to all clinical directors of dental services in Scotland. The findings indicated that not only did 'CDGA dental assessment' services need to be further defined but that they varied considerably between regions and therefore required further investigation.

A semi-structured telephone interview was developed to question the clinical director in each Scottish CDS $(n=18)$ and HDS $(n=3)$, or their nominated representative. All health boards were represented with a range of rural and urban locations.

The following questions formed the semi-structured interview regarding the assessment:

- Is the service for assessment of children prior to CDGA a 'dedicated' service, part of normal clinics or an accident and emergency (A\&tE) service?

- How soon after the pre-CDGA assessment does the child's CDGA visit take place?

- What grade of staff member carries out the assessment, and are these specialist paediatric dentists?

- What information is obtained during the assessment?

- How long does the assessment take?

- When there is a treatment plan specified in the referral letter, how often is this changed with respect to the teeth to be extracted, and the anaesthetic to be used?

- If the treatment plan is changed, are there likely to be more or less extractions?

- What alternative treatments, to the one specified in the referral treatment plan, are available on site?

All of the questions were sent in writing to the interviewee in advance of the interview to ensure the availability of the information and to reduce inaccuracy due to misinterpretation. The telephone responses were noted by a research assistant (CT) and were subsequently sent back to each interviewee in writing to confirm their validity and accuracy. This was done twice: firstly with only their own details, and secondly including all the other responses which had been made anonymous. The interviews were carried out between June and December 2001 (CT) and then updated and revalidated in May 2002 by telephone (MTH).

Interviewees were asked if their centre or area recommended that a standardised letter or form be used by primary care practitioners when referring children for CDGA in the secondary centres. Where one was used regularly, interviewees were asked to send a copy.

\section{RESULTS}

Responses were obtained from 21 individuals, mostly clinical directors from the CDS or HDS, but in three cases by a nominated member of their dental team who had direct working experience of the assessment system. The interviews took between 15 and 40 minutes, depending on the complexity of the assessment procedures. Following receipt of their responses, two interviewees made minor amendments.

\section{CDGA assessment services}

Ten interviewees indicated that they had a dedicated CDGA assessment service for children, with one of these stating that this would newly come into effect in June 2002. Of the other 11 interviewees, one indicated that their dedicated service applied only to children over 8 years when permanent teeth were to be extracted. One respondent reported there was a 'dedicated' assessment for some of the cases within their area due to a separation of the CDS and GDS CDGA pathways. Nine interviewees stated that assessment services prior to CDGA were not separate from normal clinics. Indeed, one was incorporated into a dental hospital A\&tE service.
When a written referral specifying CDGA was received, 8 interviewees indicated that the assessment would occur on the same day as the child's extractions, and 11 on a different day (ranging from 6 to 7 days later). However, 5 of the 11 individuals included provisos, for example the age of the patient might determine a same-day CDGA. Two respondents could not specify whether it would be on the same or a different day. The provision of sameday extractions was particularly common, although not exclusive to the more rural locations.

Sixteen services would accept a CDGA referral that did not have a detailed treatment plan. Seven interviewees stated that an assessment appointment would be sent to coincide with the CDGA appointment, dependent on the medical status of the child. Nine individuals indicated that the assessment appointment would be discrete from the CDGA (ranging from 10 to 60 days later), and of these, three stated that this depended on circumstances, such as age and location.

When a child attended without a written referral (1 respondent indicated that this was rare) 12 respondents indicated that the patient would be provided with an assessment service. Nine services would not progress with treatment unless a written referral was provided.

\section{The CDGA assessors}

As shown in Figure 1, a range of grades of staff carried out the CDGA assessment. Grades incorporated into the 'other' category were each cited once and included: chief administrative officer, receiving dentist, house officer/general professional trainee, senior house officer, specialist registrar and consultant: with the last four cited by one of the HDS interviewees only. In five areas there was one grade of staff involved in the assessment, in eight there were two grades, in seven there were three grades involved and in one area there were four grades.

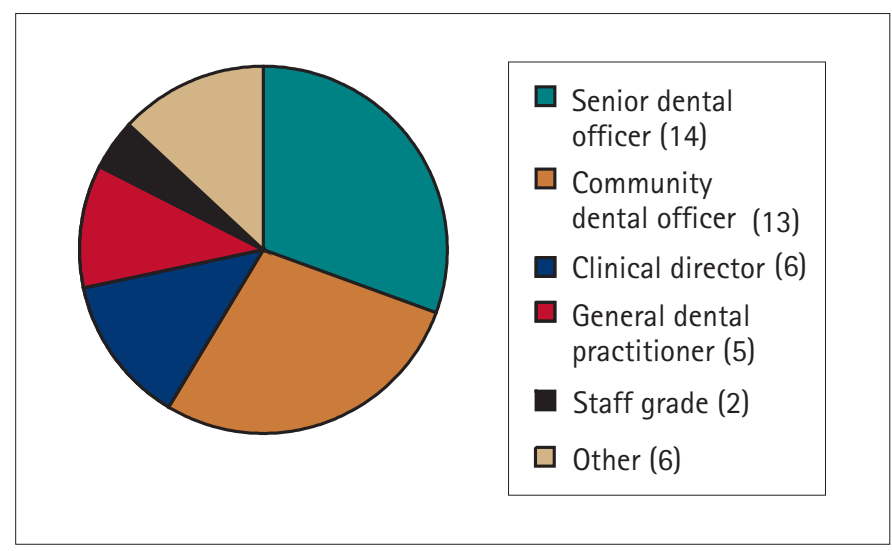

Fig. 1 Number of interviewees who quoted the number and grade of staff member carrying out assessments prior to CDGA in their area

Three of the interviewees indicated that the assessors were specialist paediatric dentistry staff, and a further four indicated that some of their team members were on either the surgical dentistry or the paediatric dentistry specialist list. Fourteen reported that none were specialist paediatric dentists. Despite this, 16 interviewees indicated that they would describe their staff team as 'skilled and trained in the treatment of children'. Four interviewees said that they considered their staff members 'skilled but not specifically trained' in paediatric dentistry.

\section{Information obtained during assessment}

The information obtained during the assessment is shown in Figure 2. Less than half of interviewees $(n=9)$ stated that alternative treatment options would be discussed at the CDGA assess- 


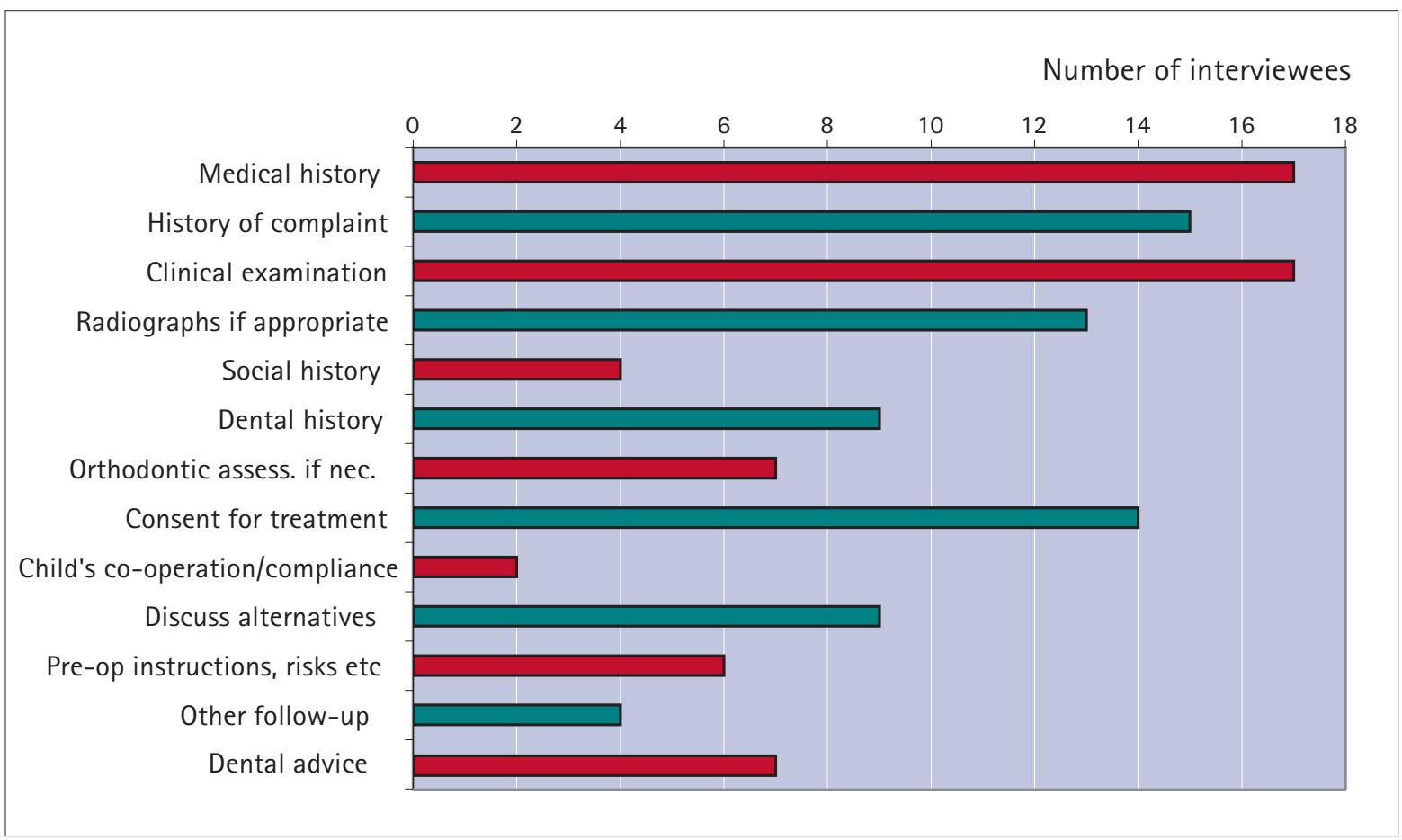

ment. Some respondents reported that referring practitioners in some areas would have already explored these alternatives. Fourteen interviewees indicated that consent for the procedure would be obtained as part of the assessment. Four individuals noted that some information eg medical history, social history and radiographs would be provided by the referring dentist.

Almost all respondents indicated that the duration of the assessment was variable and dependent on the patient. However, when further prompted, they gave an estimated range of between 2 minutes to 60 minutes (one individual was unable to specify a time). Overall the mean value was: 14 minutes (SD 7.5 minutes). Where a range was indicated, the mid-point of the range was used for this calculation.

\section{Changes to the treatment plan on the referral letter}

Interviewees were asked to estimate the regularity with which the treatment plan provided in the referral letter was changed following the assessment in terms of: a) the type of anaesthetic to be used and b) the number of teeth to be extracted. The responses are shown in Table 1. The findings indicated that the number of teeth to be extracted was changed more regularly than the anaesthetic to be used.

Table 1 Number of clinical directors who considered the assessments which lead to a change in the treatment plan

\begin{tabular}{l|c|c|c}
\hline Changes to treatment plan & Rarely/never & Sometimes & Often \\
\hline Anaesthetic used & 10 & 9 & 2 \\
\hline Number of teeth & 5 & 8 & 8 \\
- More teeth & 19 & & \\
- Less teeth & 0 & & \\
- Neither/could not say & 2 & & \\
\hline
\end{tabular}

These responses were further explored according to whether or not the interviewee had indicated that the assessment would be on a different day from the child's extractions. This revealed a greater number of interviewees who indicated that changes to the treatment plan occurred 'often' or 'sometimes' among the 'different day' assessments when compared with the 'same day' assessments.

\section{Availability of alternatives to CDGA on the CDGA sites}

Thirteen interviewees indicated that nitrous oxide inhalation sedation (IHS) was an alternative to GA in their area, although two noted that it was not available at all sites. Hypnosis and behaviour management were each cited by three individuals, with virtual reality, 'time and patience' and 'TLC' each cited by one individual. One of the two respondents who reported that there was no alternative available added that these would have been attempted by the time a child was referred for a CDGA. The range of responses received is shown in Figure 3.

\section{Referral forms}

Fourteen clinical directors submitted the standard referral form used by primary practitioners in their area. These forms ranged from one page of A4, used for generic assessment referral, through to five different forms with three information sheets for different aspects of the patient's referral, eg day case admission, anaesthesia implications, medical history, post-operative instructions. This study did not quantify the regularity with which these forms were used.

Where a medical history was recorded, a range of prompts was given, from an unstructured space to a checklist of 10 to more than 20 questions. Three of the forms recorded whether or not the dentist had checked the child's medical history. Ten forms included the justification for the choice of the anaesthetic, sometimes with a choice of prompt boxes, eg special needs, infection, behavioural, multiple extractions/oral surgery.

\section{DISCUSSION}

In Scotland, the CDS is the major provider of CDGA services. One third of the CDS clinical directors' respondents were directly involved in their CDGA service. Three others nominated a respondent whom they felt was best able to provide the most accurate information. Sending the questionnaires to the respondents prior to the telephone interview enabled them to collect the required information before data collection. This was particularly important for those not participating directly in the service provision. The principal researcher (CT) was a non-dentist and the individual responses were twice verified by each individual respondent and then further updated and revalidated by a dental researcher (MTH). 
Fig. 3 Number of interviewees who cited each alternative treatment as being available on the CDGA assessment site in their area/centre

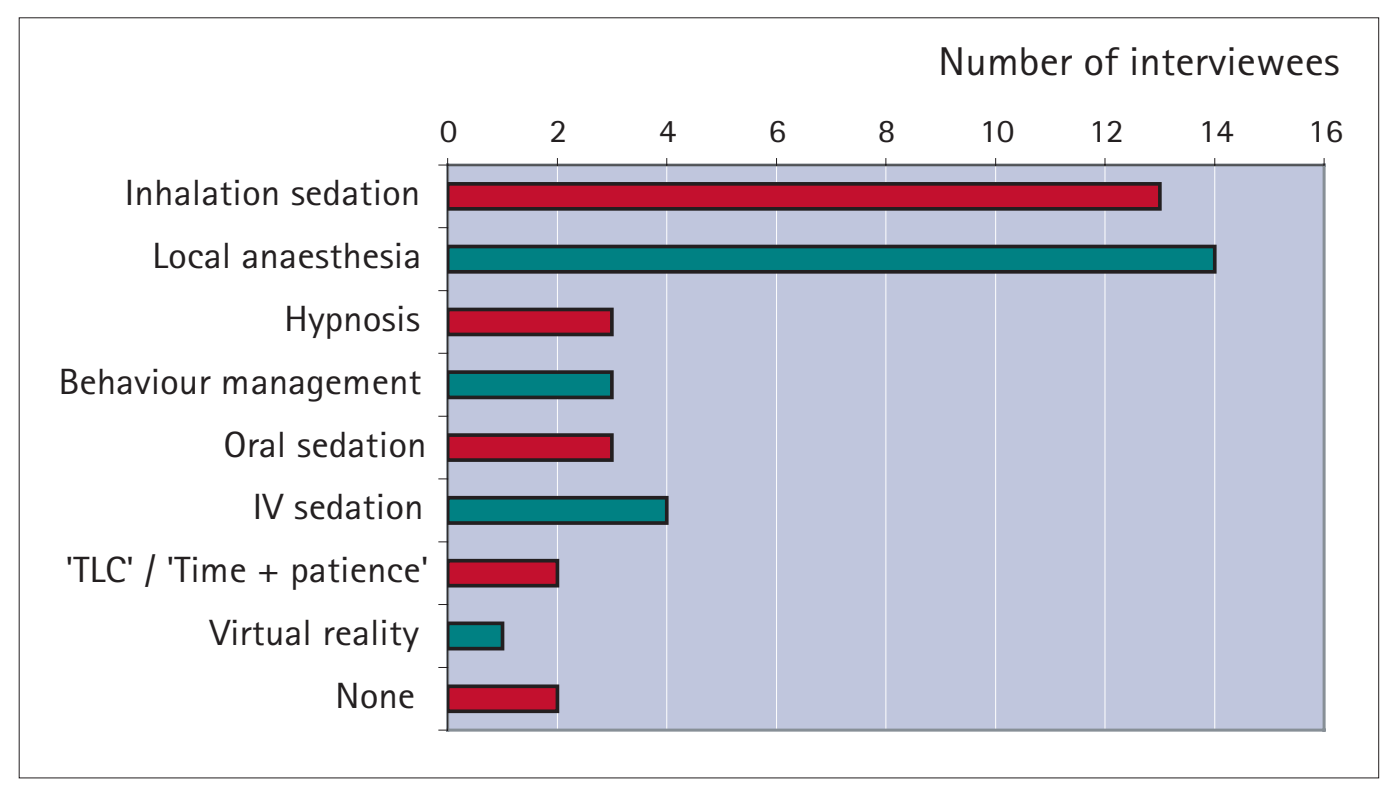

In this way it was hoped that both the questioning and the answers provided were as accurate and valid as possible but bias and ambiguity cannot be completely ruled out.

This study was designed to collect qualitative information on the current provision of pre-CDGA dental assessment services in Scotland, thus data such as waiting times were estimated by the respondents. More robust information concerning waiting times within the CDGA pathway of care in each area would have required quantitative data collection and was outside the remit of this investigation. The results of this study must therefore be interpreted accordingly.

Assessment of children before CDGA extraction occurred in some form in all areas. There was considerable structural variation in the dental assessment process in respect to duration, information obtained and the availability of alternative treatments. One determining factor was the geography of the area, and the distance over which children had had to travel to access services. This study did not attempt to quantify the number of child patients involved in each region but there is undoubtedly considerable variation in demand for this service and this will have had an impact on both the level of service provision and availability of trained staff.

Whenever the assessment and the CDGA visit are closely timed, the likelihood of offering an alternative to CDGA will reduce, since parents will already have prepared their child for a general anaesthetic and the CDGA appointment may even have already been booked. Despite this, we found that, for over a third of the services, the assessment occurs on the same day as the CDGA extraction. However, in many outlying or rural areas of Scotland, there are significant distances involved for patients travelling to CDGA centres. This must be taken into account if CDGA guidelines are developed. Nevertheless, the number of children who need to travel long distances for CDGA extractions may be further reduced by the better provision of local assessment services and improved availability of alternative treatments, provided links with anaesthetic colleagues for determination of 'fitness for general anaesthesia' are maintained. Indeed, one of the difficulties encountered by the researchers in this study was disentangling the 'dental' from the 'anaesthetic' assessment. This may be the reason for the wide variation in the time needed for the assessment between centres ( 2 minutes to an hour). The availability and time taken for other investigations such as radiographs could also form part of the explanation. Nevertheless, there are two relevant medical parallels that deserve consideration. Firstly, accessing paediatric medical treatment under general anaesthesia in rural parts of Scotland also often involves long-distance travel. Secondly, walking in from the street, with a letter from a primary care provider for immediate and direct access to a general anaesthetic service without a further specialist assessment, is virtually unheard of in medicine.

The majority of interviewees considered the assessors to be 'skilled and trained' in the treatment of children, as recommended by the Scottish Executive Health Department guidelines (2001). ${ }^{1}$ However, both the respondents' and the health department's definition of these terms are unclear, and there remains uncertainty and differences of opinion concerning whether this should mean inclusion on the General Dental Council's specialist paediatric dentistry list.

Tyrer reported that if parents and children are 'actively discouraged' from having a CDGA at the pre-anaesthetic assessment, $75 \%$ can be successfully treated without it. However, over $20 \%$ of the children in that study were referred for orthodontic extractions. ${ }^{8}$ In the present study many of the assessment clinics did not include consideration of alternatives to CDGA, with some respondents assuming that the referring practitioner would already have attempted this. Consideration and provision of such alternatives may, however, be more appropriately carried out at dedicated assessment clinics. While IHS was cited as an alternative to GA by 13 interviewees, it appears that not all regularly go on to use this treatment modality in children's extractions. Where the number of teeth to be extracted was said to change following assessment, almost everyone indicated that the number would tend to increase. This is consistent with published work which suggests that it reduces the likelihood of the need for a repeat GA in the future. ${ }^{4,9}$

It has been suggested that it may be appropriate to routinely refer children to the care of a service where the treatment plan can be developed by specialist staff, who could also offer full oral rehabilitation and not just extractions. ${ }^{9}$ Thus, when considering the future of CDGA services in Scotland, the potential for the development of links between primary care practitioners and specialist paediatric teams in the community, including professionals complementary to dentistry, may be worth exploring.

There were local standardised referral forms issued by the majority of CDGA service providers but it is not known how consistently they were used. They varied considerably in content and length, meriting consideration for future guidelines. Indeed, the study showed that information provided by primary care practitioners to CDGA assessors was lacking in many respects, especially where discussion of alternative treatment modalities and the justification for the CDGA referral were concerned, and clarifying the 
extent to which children and parents were involved in the initial decision-making process, prior to obtaining informed consent.

Further study to determine the remit and the clinical effectiveness of the dental assessment prior to CDGA is required and the development of national guidelines in this area would be of value.

\section{CONCLUSION}

There is considerable variation in the referral and assessment of children referred for CDGA extractions in Scotland. To some extent, this reflects geographic limitations and local need. The development of guidelines in this area may help to both define and clarify the purpose of the dental component of the CDGA assessment, its timing in relation to both the CDGA visit and to the provision of alternative treatments, and the qualification and training of assessors. National agreement on the adoption of more standard referral forms may reduce the variation in CDGA assessment across Scotland.
This study was supported by the Scottish Executive: CEPS Project Number: CA99/42

1. Guidance on general anaesthesia and sedation for dental treatment. Edinburgh: Scottish Executive Health Department, 2001. NHS HDL 29, 2001.

2. Maintaining standards. Guidance to dentists on professional and personal conduct General Dental Council, 1997: modified May 1998.

3. Landes D P, Clayton-Smith A J. The role of a pre-general anaesthetic assessment for patients referred by general dental practitioners to the community dental service. Community Dent Health 1996: 13: 169-171.

4. Grant S M B, Davidson LE, Livesey S. Trends in exodontia under general anaesthesia at a dental teaching hospital. Br Dent J 1998; 185:347-352

5. Holt R D, Al Lamki S, Bedi R, Dowey J A, Gilthorpe M. Provision of dental general anaesthesia for extractions in child patients at two centres. Br Dent J 1999; 187: 498-501.

6. Burns M T, Blinkhorn AS, Asbury A J. An evaluation of the behaviour of children undergoing dental extraction under general anaesthesia. Anaesthesia 1992; 47: 1073-1074.

7. McCormac C, Kinirons M. Reasons for referral of children to a general anaesthetic service in Northern Ireland. Int J Paediatr Dent 1998; 8: 191-196.

8. Tyrer $G$ L. Referrals for dental general anaesthetics - how many really need GA? BrDent J 1999; 187: 440-444.

9. Harrison M, Nutting L. Repeat general anaesthesia for paediatric dentistry. Br Dent」 2000; 189: 35-37. 